\title{
Transposons: cut-and-paste gene delivery
}

\section{From mutagenesis to gene therapy for hemophilia, transposons - mobile genetic elements - have proven themselves innovative tools in the laboratory and the clinic. Caitlin Smith takes a look at some present offerings of transposon products and the promise of applications.}

Gene therapy, the therapeutic culmination of years of research on gene targeting and delivery, involves the ability to deliver a gene to the relevant organ so that it can correct a naturally corrupted genetic function. The technical difficulties of such achievement are not limited to therapeutic promises. The delivery of exogenous genetic material, by no means easy despite recent advances, is an invaluable tool for researchers needing to introduce genes into cell lines, tissues in culture or even whole animals for research purposes.

Research tools and therapeutic promises are evolving in parallel, and although gene therapy cannot yet be considered as routine clinical procedure, gene delivery and targeting in experimental systems is a ubiquitous tool in many research labs.

\section{Jumping in the tool box}

Among the most important methods used for gene delivery are viral vectors, which take advantage of the natural ability of viruses to infect a host's cells, and RNA transfection, a newer tool that permits the delivery of mRNA or small noncoding RNAs. Many companies have invested in the development and refinement of such tools for gene delivery, and researchers have a panoply of reagents at their disposal. In addition to these reagents, researchers and companies alike have recently turned toward another promising tool: mobile genetic elements called transposons.

Transposons, also known as jumping genes, are sequences of DNA that can move from one site in a genome to another by the process of transposition - the 1983 Nobel prize-winning work of Barbara McClintock. Transposition has the potential to wreak havoc in a genome: it can result in lethal mutations and can alter the amount of DNA in the genome. Yet surprisingly, we apparently have learned to live with it. The human genome contains more than 3 million copies of transposons, which comprise about $45 \%$ of our total DNA, with each transposon copy separated by only 500 nucleotides on average. Certain transposons, however, can cause diseases, including hemophilia A and B, severe combined immunodeficiency, porphyria, predisposition to cancer and Duchenne muscular dystrophy.

Researchers' abilities to manipulate transposons, to use them as tools for gene insertion and delivery, is steadily growing. One well-known family of Drosophila melanogaster transposons, called $\mathrm{P}$ elements, appeared in the species in the middle of the twentieth century and spread through every D. melanogaster population within 50 years. These have proven useful to researchers because $\mathrm{P}$ elements can be injected into embryos to insert genes into D. melanogaster.

The occurrence of transposons in many different organisms is an exciting aspect of their usefulness as research tools. According to David Largaespada, associate professor in the department of genetics, cell biology and

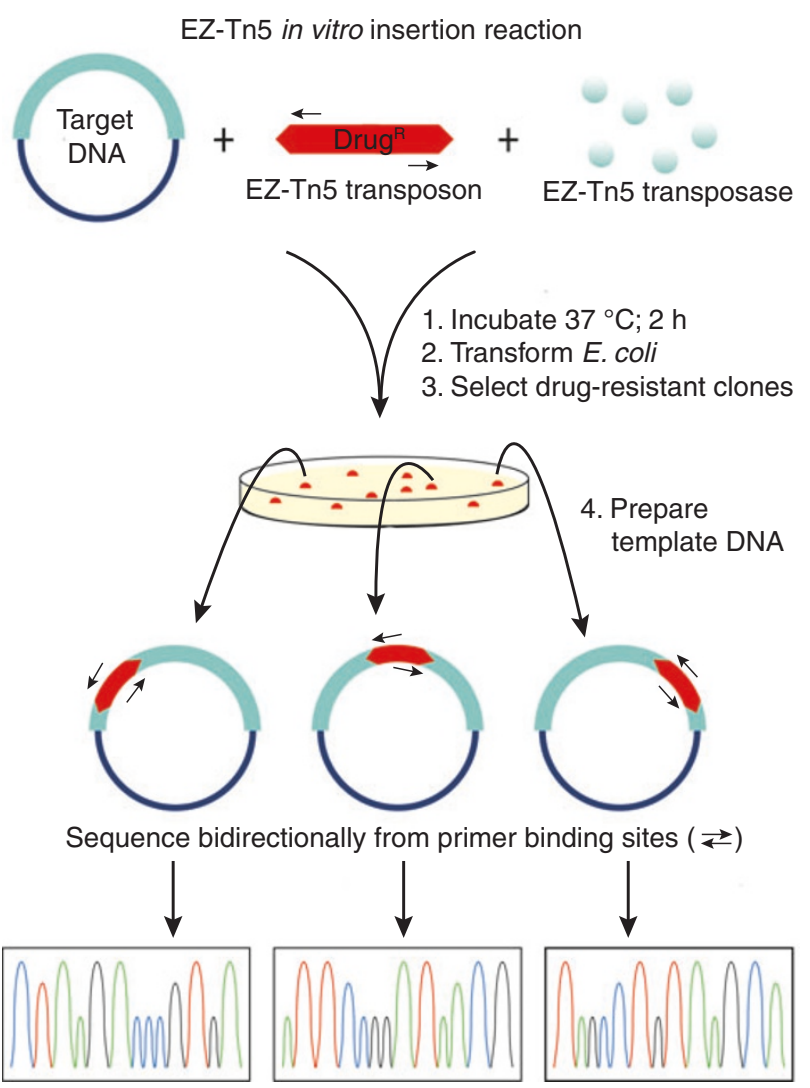

The process for generating DNA sequencing template using an Ez-Tn5 Insertion kit. (Courtesy of Epicentre Biotechnologies.) 
development, and the Cancer Center at the University of Minnesota, this characteristic of transposons has allowed a greater variety of species to be used as model systems for genetic studies. "If we look at the past, transposons have been used to do forward genetic screens and to make comprehensive insertion mutation libraries in many species," says Largaespada. "Now, it is possible to consider similar projects in vertebrate species such as Xenopus, zebrafish, rat and perhaps species we haven't yet considered as models." Largaespada also thinks that the usefulness for geneticists is being enhanced by the availability of commercial products, such as kits, which are designed specifically for these types of projects, at least in microbial genetics. "The commercial products out there have brought real genetics to species that weren't used this way before, [and] the commercialization of transposons for applications in vertebrates is likely to come soon."

\section{Off-the-shelf transposition}

Several companies offer transposon-based kits for just these types of projects. Finnzymes, for example, offers their Finnzymes Transposon Tool product line, based on the in vitro transposition reaction of the bacteriophage $\mathrm{Mu}$, a common transposon tool. These tools are packaged into three separate transposonbased kits that suit different applications: the Template Generation System II introduces primer binding sites randomly into foreign target DNA, the Mutation Generation System generates insertion mutation libraries from any target gene, and the Stop Generation System inserts translational stop codons into any target gene for generation of C-terminally truncated proteins (especially useful for functional analyses of proteins). Each kit contains MuA Transposase enzyme, artificial transposons (Entranceposons) with antibiotic resistance marker genes, and all the necessary buffers, primers and control reagents.

According to Ville Tieaho, research scientist and group leader at Finnzymes, the latter kit is unique among transposon-based products: "The Stop Generation System includes proprietary modifications in the Entranceposon DNA sequence. The modifications are made in the very terminal regions of the Entranceposon DNA, which usually does not tolerate sequence changes. This kind of product would be nearly impossible to make with the competing transposition systems."

Tieaho explains that the central mechanism behind their transposon kits is the
Stop Generation System Kit from Finnzymes. MuA transposase catalyzes the in vitro transposition reaction and randomly inserts Entranceposons into target DNA plasmids (top). E. coli transformed with the plasmids produce a library of truncated proteins (bottom). (Courtesy of Finnzymes.)

formation of a tetramer of MuA transposase around the terminal sequences of a linear Entranceposon DNA molecule. The activated transposon-complex finds and makes a 5-base-pair staggered cut in the target DNA, and then the Entranceposon DNA is inserted into the target site. One advantage of kits that use the enzyme MuA transposase, such as those from Finnzymes and other manufacturers, is that the MuA-catalyzed in vitro transposition is essentially random, so there are no hot-spots for insertion. As Tieaho explains, "Some other in vitro transposition systems tend to favor, for instance, $(\mathrm{A}+\mathrm{T})$ rich areas in target DNA over $(\mathrm{G}+\mathrm{C})$-rich areas."

Like the Finnzyme Template Generation System II, Invitrogen also offers a kit aimed at DNA sequencers. Their GeneJumper Kit is designed to insert primer binding sites randomly into target DNA in preparation for sequencing. Each kit includes the GeneJumper Transposon with chloramphenicol or kanamycin resistance, $\mathrm{MuA}$ Transposase, reaction solutions, PCR and sequencing primers, control template and primers, One Shot competent Escherichia coli, SOC medium and a control plasmid.

New England BioLabs' GPS-1 Genome Priming System is another such kit for researchers who want to generate a population of DNA sequencing templates with randomly interspersed primer-binding sites. GPS-1 is a transposon-based in vitro system that uses the TnsABC Transposase to insert a transposon (NEB's Transprimer) randomly into the target DNA. The GPS-1 System also includes two Transprimer transposons, encoding kanamycin or chloramphenicol resistance.

Epicentre offers too wide a selection of transposon-based products to detail here, but they generally fall under the headings of the Epicentre EZ-Tn5 Transposon Systems and HyperMu Transposon Systems, which are designed for both in vitro and in vivo applications. Among their many offerings are kits and products for sequencing applications like others, but also for making unidirectional truncations of a protein encoded by any sequence, mapping protein domains
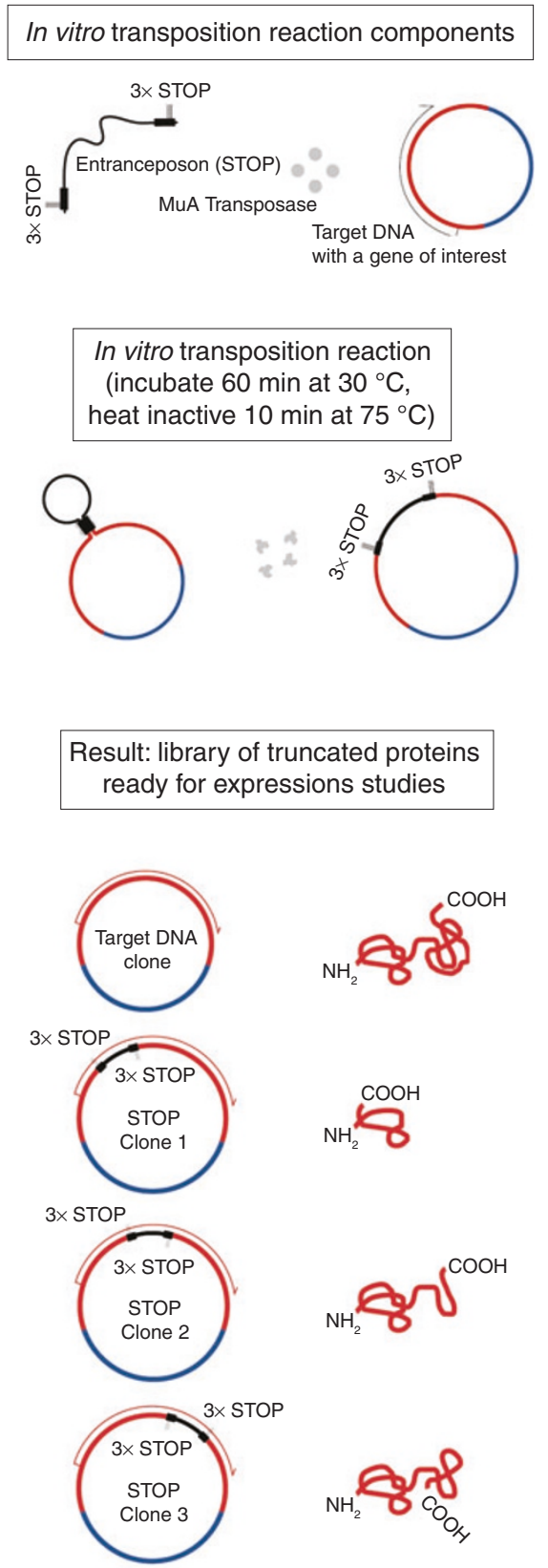

by in-frame transposon insertion, and making truncations of protein-coding sequences cloned in deletion vectors.

Whichever kits or products you choose, heed some sage advice if you are a novice gene jumper: don't jump right into your new kit with your most precious samples of genetic material, no matter how straightforward the new product appears. William Reznikoff, professor of biochemistry at the University of Wisconsin-Madison, studies the basic molecular mechanisms of transposition, and uses transposons for genomic analyses. He believes that most transposonbased kits are generally good tools, but he cautions, "My primary advice to new users 
is to start out using transposons in control experiments, say in E. coli, until the user feels comfortable; after a comfort level is reached, the user should switch to using the transposons in important experiments."

\section{The therapeutic horizon}

A key component in gene therapy is gene delivery: how to get that new and improved gene in the patient's cells, where it will be active, and to keep it there, so it can remain active. Viral delivery has looked promising since the early days of gene therapy because viruses have evolved to enter host cells and strategically place their genetic material where it will be maintained, such as integrated into the host genome. But getting into a cell's genome is not the only challenge-one needs to get the transgene into the right spot. Most viruses, however, have not evolved to be particularly selective, and relying on viruses carries the risk of activating cancer-causing genes by insertional activation. In addition, the patient's defenses against infection do not distinguish between 'bad' viruses and 'good' viruses used to deliver a therapeutic gene.

In the area of gene therapy also, researchers have looked at transposons to try to circumvent these problems. When companies like Discovery Genomics started to develop transposon technology as a method of gene delivery, it seemed that this new technology would soon be on its way to the clinic, but of course there are always more challenges than expected. Nevertheless, major progresses have been made.

50 The founders of Discovery Genomics had developed a transposon called Sleeping Beauty, which is known for its efficiency in vertebrates. Largaespada, cofounder of and advisor to Discovery Genomics, says that "we started using 'cut-and-paste' transposons like Sleeping Beauty about 6 years ago as a more versatile insertional mutagen than retroviruses to find cancer genes." At Discovery Genomics, they can create mice in which Sleeping Beauty transposons are mobilized in various tissues, which causes insertion mutations affecting endogenous genes, so that solid tumors result. Largaespada's academic group is now performing forward genetic screens for various types of cancer including prostate, gastrointestinal tract, lung, and head and neck cancer. As Largaespada explains, “This hasn't been possible before, in part, because no 'cut-andpaste' transposable elements had been found or created that worked in vertebrate cells."

\section{Transposons for the lab menagerie}

Largaespada is also using transposons for long-term delivery and expression of transgenes to the soma of adult mice. "We've done this as a prelude to human gene therapy or to deliver genes we think can cause cancer to mouse somatic cells in situ," he says. His group observes that several different transposons appear to be able to deliver genes into the germline and soma of a variety of vertebrate species. Largaespada reckons that Sleeping Beauty, originally used in mice, could probably be applied to rat, hamster or other species. In fact, depending on your project, a transposon other than Sleeping Beauty, and/or a species other than mouse, may give more optimal results. "For example, the right rat strain can be induced to develop mammary cancer that is similar to human mammary cancer in that it is estrogen-dependent, unlike the situation in mouse," he says. Among the most appealing projects he cites are the induction of mammary cancer by estrogen and transposon mutagenesis in the rat. "As another example,' he adds, "the Tol2 transposon system can deliver larger transgenes than Sleeping Beauty can, and so it is better for some applications."

Though progress is promising, there are still some obstacles to overcome. "From my point of view, the biggest challenge we face is to improve the rate of germline transposition to the point where genomewide forward genetic screens can yield mutants nearly as efficiently as does the chemical mutagen ethylnitrosourea," says Largaespada. "At present, we can use transposons to make germline mutations, but the types of screens we can employ are fairly general. We need a higher rate of mutation to be able to do enhancer and suppressor type screens."

Largaespada notes too that like every biological tool, transposons are not perfect. His group's recent work shows that Sleeping Beauty transposition can create large chromosomal deletions and inversions. "So care must be taken in assigning a given phenotype to a specific transposon insertion mutation," he says. "Transposons are tools that can be part of a complementary set of approaches for functional genomics. More tools should yield better science."

Caitlin Smith is a freelance writer in Portland, Oregon (caitlin.smith@ comcast.net). 


\section{SUPPLIERS GUIDE: COMPANIES OFFERING GENE DELIVERY AND TARGETING PRODUCTS}

\section{Company}

Amaxa

Ambion

Amersham Biosciences (part of GE Healthcare)

Avanti Polar Lipids Inc.

B-Bridge International Inc

Bender MedSystems

Bio-Rad Laboratories

BTX Molecular Delivery Systems

Clontech (BD Biosciences)

Dharmacon, Inc.

Epicentre

Eppendorf

Eurogentech

Fermentas

Finnzymes

GeneChoice, Inc.

Geneflow Ltd.

Genlatis

Imgenex

Invitrogen

InvivoGen

Midwest Scientific

Microbix

Mirus

MoBiTec Molecular Biotechnologies

Molecula

MP Biology

Oz Biosciences

Polyplus Transfection

Pepscan Systems

PCI Biotech

PGC Scientific

Promega

Qbiogene Inc.

Qiagen

Roche Applied Science

Sigma-Aldrich

Stratagene

System Biosciences

Targeting Systems

Thermo Electron Corporation

Tritech Research Inc.

Upstate

Wako Chemicals SA

\section{Web address}

http://www.amaxa.com/

http://www.ambion.com

http://www4.amershambiosciences.com

http://www.avantilipids.com/

http://www.b-bridge.com/eng/index.htm

http://www.bendermedsystems.com/

http://www.bio-rad.com

http://www.btxonline.com/

http://www.clontech.com/clontech/

http://www.dharmacon.com/

http://www.epibio.com

http://www.eppendorf.com

http://usa.eurogentec.com/code/en/hp.asp

http://www.fermentas.com/

http://www.finnzymes.com/

http://www.genechoiceinc.com/

http://www.geneflow.co.uk

http://www.genlantis.com/

http://www.imgenex.com

http://www.invitrogen.com/

http://www.invivogen.com/

http://midsci.com/

http://www.microbix.com/products/04.html

http://www.mirusbio.com/

http://www.mobitec.de/mobitec_us/index.html

http://www.molecula.com/

http://www.mpbio.com

http://www.ozbiosciences.com/English/default.html

http://www.polyplus-transfection.com/EN/index.php

http://www.pepscan.nl

http://www.pcibiotech.com/

http://www.pgcsci.com

http://www.promega.com/

http://www.adenovirus.com/

http://www1.qiagen.com/

http://www.roche-applied-science.com/

http://www.sigmaaldrich.com/

http://www.stratagene.com/homepage/

http://www.systembio.com

http://www.targetingsystems.com/

http://www.thermo.com

http://www.tritechresearch.com/cgi-bin/shop/

http://www.upstate.com

http://www.wakousa.com/ 


\section{Erratum: Transposons: cut-and-paste gene delivery}

\section{Caitlin Smith}

Nat. Methods 4, 183-186 (2007).

In the version of this article originally published, the statement that "At Discovery Genomics, they can create mice in which Sleeping Beauty transposons are mobilized in various tissues, which causes insertion mutations affecting endogenous genes, so that solid tumors result" was misleading. This work was done in David Largaespada's academic lab, not at Discovery Genomics.

Also, the statement that "Sleeping Beauty transposition can create large chromosomal deletions and inversions" was incorrect. The article should have said that in some instances, Sleeping Beauty transposition can create large chromosomal deletions and inversions, though in many cases does not.

In the Suppliers guide, the listing for Clontech (BD Bioscience) should be Clontech (Takara Bio). 